Gut, 1986, 27, 1006-1013

\title{
Human colonic smooth muscle: spontaneous contractile activity and response to stretch
}

\author{
R C GILL, K R COTE, K L BOWES, AND Y J KINGMA \\ From the Departments of Surgery and Electrical Engineering, University of Alberta, Edmonton, Alberta, \\ Canada
}

SUMmaRY The length dependence of the spontaneous contractile activity of human colonic muscle was assessed in vitro. Muscle obtained from the right colon was more distensible than that of the left colon. This was true for all muscle layers. Maximum spontaneous active stress was exerted by both circular and longitudinal muscle layers of the right colon at greater degrees of stretch $(p<0.001)$ than those of the left colon. The contractile frequency of longitudinally oriented strips increased with length. The contractile frequency of intertaenial longitudinally oriented strips from the right colon was lower $(p<0.001)$ than that of strips from the left colon. The contractile frequency of circularly-oriented strips from the right colon $(6 \cdot 25 \pm 0 \cdot 38 \mathrm{~min})$ was higher $(p<0.001)$ than that of strips from the left colon $(3.35 \pm 0.35 \mathrm{~min})$. The human colon appears to consist of two distinct areas based on the mechanical behaviour of the smooth muscle during spontaneous contraction.

The human large intestine may be subdivided into right and left parts on the basis of differing embryology, blood supply, innervation and function. $^{12}$ The right colon has a greater diameter than that of the left, both on abdominal radiographs ${ }^{3}$ and post-mortem, ${ }^{4}$ and also appears more distensible than the left, being the area of greatest distension in the face of a distal colonic obstruction. Colonic pathology may also show a particular pattern of localisation. Perforation of the colon occurs most frequently in the caecum ${ }^{5}$ whilst diverticular disease is most common in the descending and sigmoid colon. ${ }^{6}$ Diverticular disease has been associated with an abnormal response of the colonic muscle to stretch. ${ }^{78}$

In view of these observations, it would seem probable that regional variations in colonic function and pathology reflect an underlying difference in the mechanical properties of muscle along the length of the human colon. Indeed, the spontaneous contractions of human colonic muscle and their modulation by intrinsic neural discharge and stretch appear to be of considerable importance as colonic function, albeit impaired, continues after extrinsic denervation. ${ }^{9-11}$

The spontaneous contractile activity of colonic

Address for correspondence: Richard C Gill, GI Science Research Unit, The London Hospital Medical College, London E1 2AJ.

Received for publication 21 February 1986. muscle obtained from several animal species has been reported although little is known concerning the dependence of these spontaneous contractions upon muscle length. The majority of studies concerning the length dependence of colonic muscle have been conducted after the abolition of spontaneous contractions in order to free the contractile element and permit an analysis of the passive properties of the muscle. ${ }^{12-15}$ In these studies, the active contractile properties of the muscle were assessed by external stimulation either electrically, ${ }^{12-14}$ by $\mathrm{K}^{+}$depolarisation ${ }^{13}$ or by drugs such as acetylcholine or carbachol. ${ }^{13}$ A recent in vitro study, ${ }^{16}$ however, established the length dependence of taenia from guinea-pig caecum during spontaneous contraction.

As far as human taenia coli is concerned, its spontaneous contractile activity in vitro ${ }^{17-19}$ appears similar to that of the colon from the guinea pig, ${ }^{20}$ $\mathrm{pig}^{21}$ and even the non-taeniated longitudinal muscle layer of the dog. ${ }^{22}$ The length dependence of human taenia coli during spontaneous contraction is not known. The spontaneous contractile activity of the intertaenial longitudinal muscle layer of the human colon and its length-dependence have not been established. Spontaneous contractile activity of human colonic circular muscle layer in vitro is less clearly defined than that of the cat, ${ }^{23}$ dog $^{22}$ or pig. ${ }^{21}$ Contractions of the circular muscle layer of the human colon at a frequency of $2.4 \mathrm{~min}$ have been 
reported by van Merwyk and Duthie ${ }^{19}$ who concluded that the mechanical response of this muscle layer to stretch was weak.

This present, in vitro, study was initiated to establish the length dependence of the longitudinal and circular muscle layers of the human colon during spontaneous contraction. Variations in the patterns of spontaneous contraction and their modulation by stretch have been assessed in muscle strips from different parts of the colon.

\section{Methods}

TISSUE

Strips of human colonic muscle obtained at surgery were studied in vitro. Their contractile force and frequency in response to stretch were assessed.

\section{ACQUISITION}

Tissue was obtained from 22 patients undergoing partial colectomy for carcinoma. After resection of the diseased portion, a ring of colon (approximately $2.5 \mathrm{~cm}$ long) was removed from either the proximal or distal margin of the future anastomotic site; the blood supply of the segment was maintained until the moment of excision. Specimens were immediately placed in oxygenated Krebs' Ringer solution. Six specimens from ascending, five from transverse, four from descending and seven from sigmoid colon were obtained.

\section{PREPARATION}

The colonic segments were opened along the border of a taenia, carefully cleaned to prevent soiling of the muscle layers with faecal content, and pinned to the surface of a dissecting dish filled with oxygenated Krebs' Ringer solution. The mucosa and submucosa were cut off. Strips of muscle, approximately $4 \mathrm{~mm}$ wide, were cut with the long axis lying in the direction of the muscle fibres. Longitudinallyoriented strips (10-15 mm long) were obtained both from the taenia and the region between taeniae. Circularly oriented strips (8-10 $\mathrm{mm}$ long) were taken from the intertaenial region. Strips were pinned at one end to the Sylgard surface of a horizontal tissue chamber maintained at $37 \pm 1^{\circ} \mathrm{C}$ and continuously superfused with oxygenated Krebs' Ringer solution. Care was taken during this procedure to avoid stretching the preparations.

\section{RECORDING TECHNIQUE}

Contractile activity of the muscle strips was recorded with a force displacement transducer (Grass FT03). Each muscle strip was attached to a force transducer with surgical $4-0$ silk thread. Recordings were made of the contractile activity on a Beckman R511A polygraph.

\section{SOLUTIONS}

The superfusate used was a modified Krebs' Ringer solution containing the following (mM): $\mathrm{Na}^{+}, 139 \cdot 2$; $\mathrm{K}^{+}, 5 \cdot 4 ; \mathrm{Ca}^{2+}, 2 \cdot 5 ; \mathrm{Mg}^{2+}, 1 \cdot 2 ; \mathrm{Cl}^{-}, 125 \cdot 1 ; \mathrm{HCO}_{3}^{-}$, $22.0 ; \mathrm{H}_{2} \mathrm{PO}_{4}^{-}, 1 \cdot 2$; glucose, $10 \cdot 1$. When equilibrated with $95 \% \mathrm{O}_{2}, 5 \% \mathrm{CO}_{2}$ gas mixture at $37^{\circ} \mathrm{C}$, this solution had a $\mathrm{pH}$ of 7.3-7.4.

\section{EXPERIMENTAL PROTOCOL}

At, the start of each experiment, strips were mounted slack $(<0.05 \mathrm{mN}$ force) in the tissue chamber and allowed to equilibrate for a period of $30 \mathrm{~min}$. The length of the strips was then measured. Subsequently, the length of the strips was increased, stepwise, by increments of $1.0 \mathrm{~mm}$ each $15 \mathrm{~min}$. The experiment was concluded either when the muscle strip ceased to contract spontaneously or the upper limit of the force transducer was reached (approximately $250 \mathrm{mN}$ ). After the experiment, the strips were removed from the tissue chamber, firmly blotted without crushing, and weighed on a balance (Mettler B5) to the nearest $0 \cdot 1 \mathrm{mg}$.

\section{CROSS SECTIONAL AREA}

The cross sectional area (A) of each muscle strip was calculated from the equation $A=(M / p L) . f_{T}$. $M$ is the mass $(\mathrm{mg}), \mathrm{p}$ is the density $\left(\mathrm{mg} \mathrm{mm}^{-3}\right)$ and $\mathrm{L}$ is the length $(\mathrm{mm})$ of the strip determined at rest. The density of the tissue was taken as $1.056 \mathrm{mg} \mathrm{mm}^{-3}$. $^{12}$ $f_{T}$ is the proportion of the muscle strip contributed by the longitudinal (taenial and intertaenial longitudinally oriented strips) or circular (intertaenial circularly oriented strips) muscle layer. A value for $f_{T}$ was obtained from both histological and non-fixed transverse sections of unstretched muscle from all parts of the colon.

\section{ANALYSIS OF DATA}

Recordings of contractile activity were analysed visually. Three variables were assessed at each increment of length increase for each of the strips; active force, baseline force and contractile frequency. Active tension was taken to be the difference between maximum and minimum force during a cycle of spontaneous contraction. Data were obtained only from the last $10 \mathrm{~min}$ recording at each increment of length increase to allow a period of five minutes for the strip to accommodate the increase in length. This was found to be necessary as once the baseline tension of the strip was greater than zero, further increases in length produced an initial increase in baseline tension which decayed curvilinearly to a constant value. The major part of this decay was complete within two minutes. The length of the strip at which an increase in baseline tension was first recorded was measured and referred to as 
the initial length, $\mathrm{L}_{\mathrm{i}}$. Subsequent changes in the length of the strip were normalised with respect to this initial length.

The number of spontaneous contractions during the 10 minute recording period were counted and the contractile frequency determined.

The active and baseline stresses in the longitudinal axis of the muscle strip were calculated by expressing the active and baseline tensions, respectively, per unit cross sectional area of the appropriate muscle layer.

STATISTICAL ANALYSIS

Results are expressed as the mean $\pm \mathrm{SD}$, and analysed statistically using Student's $t$ test.

\section{Results}

\section{LENGTH STRESS CHARACTERISTICS}

Spontaneous contractile activity was recorded from all the 46 longitudinally-oriented strips of taenia (Fig. 1a), 40 inter-taenial longitudinally-oriented strips (Fig. 1b) and 32 inter-taenial circularlyoriented strips (Fig. 1c, d).

In general, the spontaneous active force generated increased with length up to a maximum. Further increases in length were associated with a decrease, or abolition, of active force generated and a marked increase in baseline stress (Fig. 2).

Differences were observed in the response of each muscle layer to stretch.

\section{(a) Taenial longitudinally-oriented strips}

Maximum active force generated occurred at $2 \cdot 40 \pm 0 \cdot 15 \mathrm{~L}_{\mathrm{i}}$ in taenial longitudinally-oriented strips
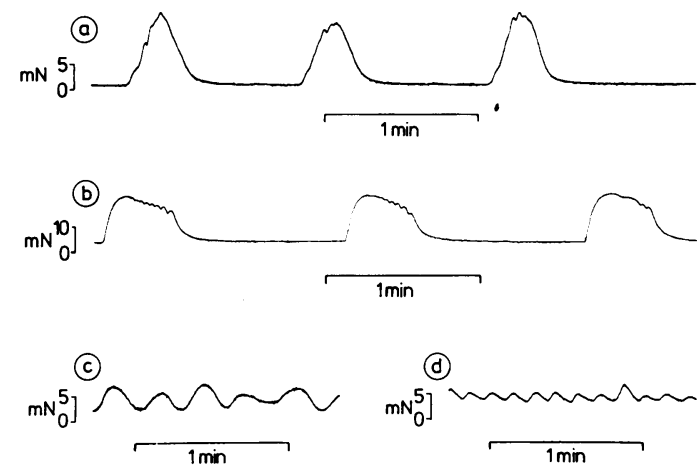

Fig. 1 Spontaneous contractile activity recorded from taenial $(A)$ and intertaenial $(B)$ longitudinal strips obtained from the transverse colon at $1 \cdot 15 L_{i}$. Note the different pattern of spontaneous contractions recorded from intertaenial circular strips obtained from the left $(C)$ and right (D) transverse colon at $2 \cdot 2 L_{i}$.
Table 1 Maximum active stress, and length at which this was observed, for longitudinal and circular muscle layers of human colon in vitro

\begin{tabular}{ccc}
\hline Colon region & $\begin{array}{l}\text { Maximum } \\
\text { active stress } \\
\left(m N m^{-2}\right)\end{array}$ & $\begin{array}{l}\text { Length at } \\
\text { maximum active } \\
\text { stress }\left(L_{i}\right)\end{array}$ \\
\hline Ascending & & \\
T L-O & $43 \pm 11$ & $2 \cdot 40 \pm 0 \cdot 15$ \\
I-T L-O $\dagger$ & $91 \pm 17$ & $2 \cdot 35 \pm 0 \cdot 15$ \\
I-T C-O & $5 \cdot 5 \pm 1 \cdot 3$ & $3 \cdot 60 \pm 0 \cdot 20$ \\
Transverse & $16 \pm 6$ & $2 \cdot 35 \pm 0 \cdot 20$ \\
T L-O & $118 \pm 29$ & $2 \cdot 35 \pm 0 \cdot 15$ \\
I-T L-O & $6 \cdot 4 \pm 0 \cdot 8$ & $3 \cdot 60 \pm 0 \cdot 40$ \\
I-T C-O & & \\
Descending & $22 \pm 7$ & $1 \cdot 80 \pm 0 \cdot 20$ \\
T L-O & $120 \pm 23$ & $1 \cdot 90 \pm 0 \cdot 15$ \\
I-T L-O & $5 \cdot 5 \pm 0 \cdot 6$ & $2 \cdot 20 \pm 0 \cdot 15$ \\
I-T C-O & & \\
Sigmoid & $23 \pm 9$ & $1 \cdot 80 \pm 0 \cdot 10$ \\
T L-O & $128 \pm 18$ & $1 \cdot 90 \pm 0 \cdot 10$ \\
I-T L-O & $11 \cdot 8 \pm 2 \cdot 0$ & $2 \cdot 15 \pm 0 \cdot 10$ \\
I-T C-O & & \\
\hline
\end{tabular}

Values are mean \pm SD.

${ }^{*}$ Taenial longitudinal muscle layer. tIntertaenial longitudinal muscle layer.

$\ddagger$ Intertaenial circular muscle layer.

obtained from the ascending and transverse - that is, right, colon, this being at a greater length $(p<0.001)$ than that of strips obtained from the descending and sigmoid - that is, left, colon $\left(1 \cdot 80 \pm 0 \cdot 15 \mathrm{~L}_{\mathrm{i}}\right)$. The maximum active force generated by the taenia of the ascending colon was greater $(p<0.001)$ than that of the taenia obtained from the transverse, descending or sigmoid colon (Table).

(b) Inter-taenial longitudinally oriented strips

Maximum active stress was recorded at $2 \cdot 35 \pm 0 \cdot 15 \mathrm{~L}_{\mathrm{i}}$ in inter-taenial longitudinally-oriented strips from the ascending and transverse colon, this being a greater length $(p<0.001)$ than that of strips from the descending and sigmoid colon $\left(1.90 \pm 0.15 \quad \mathrm{~L}_{\mathrm{i}}\right)$. These values are not significantly different $(p>0.1)$ from those of taenia obtained from corresponding regions of the human colon. The maximum active stress exerted by the inter-taenial longitudinal muscle layer did not vary significantly ( $p>0.25)$ between strips obtained from different parts of the human colon but was, in all cases, greater $(p<0.001)$ than that of the taenia (Table).

\section{(c) Circular strips}

Maximum active stress was recorded at $3 \cdot 60 \pm 0 \cdot 30 \mathrm{~L}_{\mathrm{i}}$ in inter-taenial circular strips obtained from the ascending and transverse colon, this being at a 

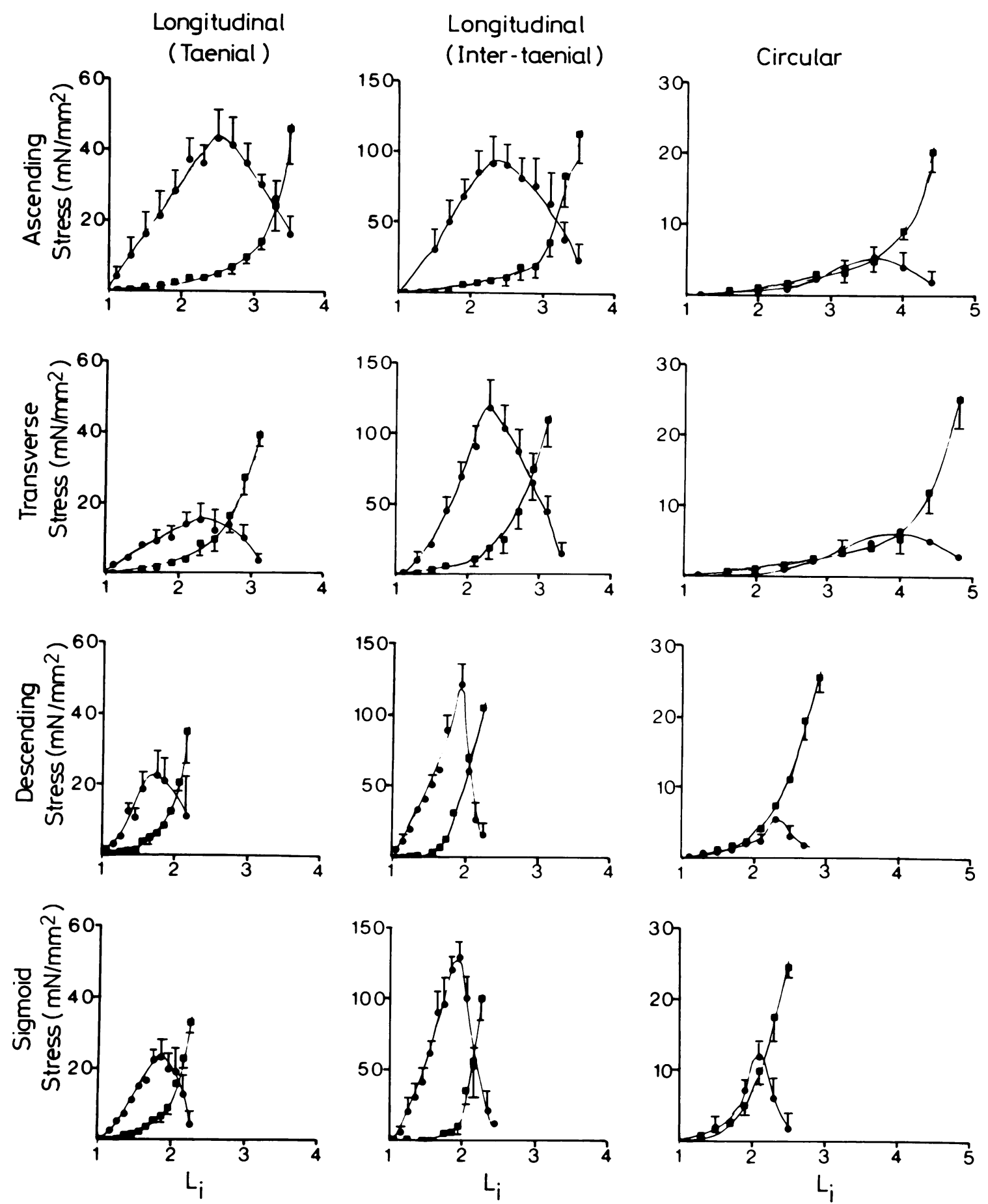

Fig. 2 Active (solid circles) and passive (solid squares) stress of spontaneously contracting longitudinal and circular muscle layers of human colon as a function of their initial length $\left(L_{i}\right)$. Standard deviations of the mean values are shown as bars except when smaller than the symbol. 
greater length $(p<0.001)$ than that of strips from the descending and sigmoid colon $\left(2 \cdot 20 \pm 0 \cdot 10 \mathrm{~L}_{\mathrm{i}}\right)$. The maximum active stress exerted by the circular muscle of the sigmoid colon was greater $(p<0.001)$ than that of the ascending, transverse or descending colon. In all parts of the colon, the active stress exerted by the circular muscle was less $(p<0.001)$ than that of either the taenial or inter-taenial longitudinal muscle (Table).

\section{LENGTH-FREQUENCY CHARACTERISTICS}

In order to compare the effect of increasing length on the contractile frequency of strips obtained from different parts of the colon, the contractile frequency has been expressed in relation to the length at which the maximum active stress was recorded, referred to as $\mathrm{L}_{\max }$.

\section{(a) Longitudinal strips}

The spontaneous contractile frequency of taenial and intertaenial longitudinal muscle obtained from all colonic regions increased with length between $0 \cdot 5-1.0 \mathrm{~L}_{\max }$ (Fig. 3a, b). The contractile frequencies of strips obtained from the ascending and transverse, ie right, colon were not significantly different $(p>0.4)$; these data are thus combined. Similarly, no significant difference $(p>0.4)$ was observed in the descending and sigmoid, ie left, colon, and these data are also combined. No consistent difference $(0.05<p>0.4)$ was observed between the contractile frequencies of taenial strips obtained from the right or left colon between $0 \cdot 5-1 \cdot 0 \mathrm{~L}_{\max }$. Further, the contractile frequencies of intertaenial longitudinal strips obtained from the left colon were not significantly different $(p>0 \cdot 1)$ from those of taenial longitudinal strips obtained from either the right or left colon at lengths of $0.5-1.0 \mathrm{~L}_{\max }$. The contractile frequencies of intertaenial longitudinal strips from the right colon were, however, lower $(p<0 \cdot 001)$ than those of either intertaenial strips from the left colon or taenia from right and left colon at all lengths studied.

\section{(b) Circular strips}

The contractile frequency of intertaenial circular strips obtained from all parts of the colon did not vary significantly $(p>0.4)$ with increases in length between $0 \cdot 3-1 \cdot 0 \quad \mathrm{~L}_{\max }$ (Fig. 3c). The contractile frequency of strips obtained from the right and left parts of the colon showed marked regional variation (Fig. 4).

Above $\mathrm{L}_{\max }$, the contractile frequency of the intertaenial circularly-oriented strips increased. This increase in frequency could not be accurately quantified as contractions were observed at frequencies of 3-7 min, 8-15 $\mathrm{min}$ and $14-27 \mathrm{~min}$; on
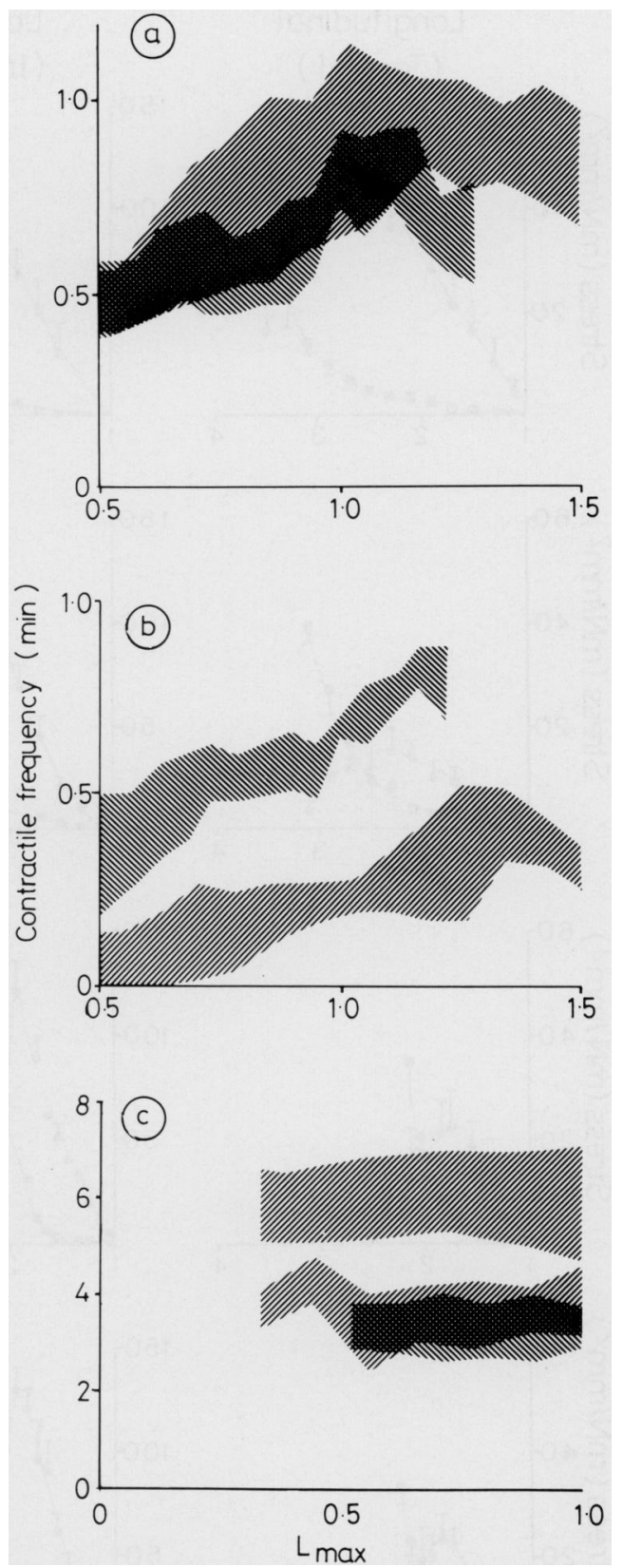

Fig. 3 Spontaneous contractile frequency of taenial $(A)$ and intertaenial $(B)$ longitudinal strips as a function of length. Shaded areas represent the mean $\pm 1 S D$ of data obtained from ascending and transverse (t) and descending and sigmoid $(\mathbb{\$})$ colon respectively. The spontaneous contractile of intertaenial circular strips $(C)$ obtained from the ascending and right-transverse (upper panel left transverse (lower panel and descending and sigmoid (W) colon are similarly expressed. 
occasions these contractions summated and gave rise to contractions of prolonged duration which sometimes recurred at a frequency of 1-3 min (Fig. $5)$.

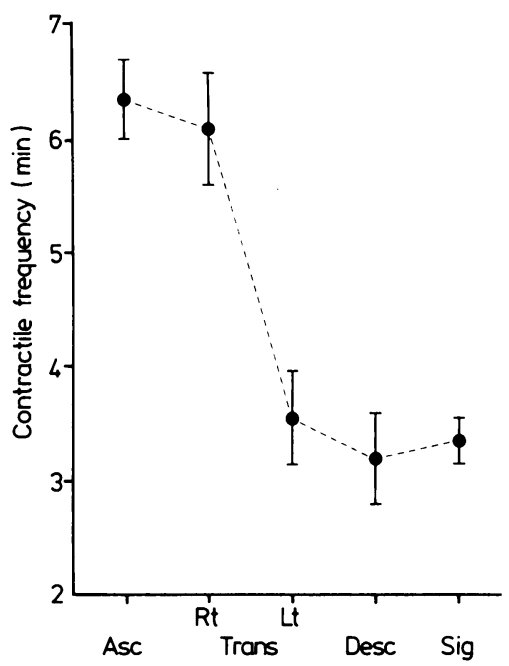

Fig. 4 Contractile frequency of circular strips obtained from different parts of the human colon at strip lengths $<L_{\max }$.
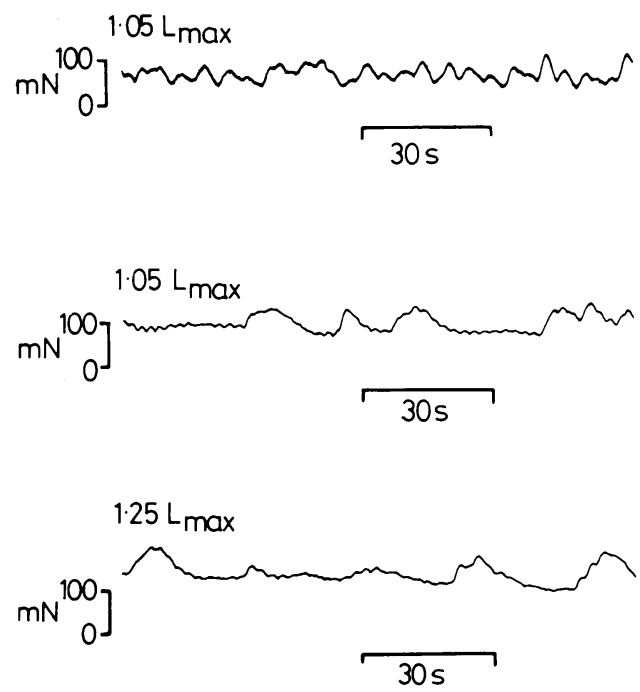

Fig. 5 Spontaneous contractile activity of intertaenial circular strip from the ascending colon at lengths exceeding $L_{\text {max }}$. Note contractile activity at frequencies of 8-15 min and 14-27 min; these could summate and give rise to contractions of long duration.

\section{Discussion}

Spontaneous contractions of both circular and longitudinal muscle layers of the human colon were, in general, only observed when the baseline stress exceeded zero at lengths equal to or slightly greater than the initial length $L_{i}$. This suggests that some degree of stretch is required in order to elicit spontaneous contractile events from human colonic isolated muscle.

The active and baseline stress curves of both circular and longitudinal muscle layers of the right (ascending and transverse) colon were less affected by length changes than were the left (descending and sigmoid) colonic muscles. This would be consistent with the right colon being more distensible than the left and functioning as an accommodative reservoir in which the temporary storage, absorption and bacterial fermentation of content could proceed. ${ }^{2}$ This marked difference between the right and left regions may be related, to their differing innervation, blood supply and embryological origin. ' A further distinction could be made between the contractile properties of the right and left colon. Circular strips obtained from the right colon contracted at nearly twice the frequency of strips from the left colon at lengths less than $\mathrm{L}_{\text {max }}$. $A$ similar observation has been made in vivo. ${ }^{24}$ This provides some evidence of a right-to-left colonic gradient.

The spontaneous active stress exerted by both circular and longitudinal muscle layers increased with length until an optimal length, $\mathrm{L}_{\max }$, was reached, and further length increases decreased the force generated. This is similar to findings reported by others in caecum ${ }^{1725}$ blood vessels, ${ }^{26} 2728$ urinary bladder, ${ }^{29}$ small intestine, ${ }^{30}$ uterus ${ }^{31}$ and colonic $^{13-15}$ smooth muscle preparations in which spontaneous contractile events have been arrested. The marked increase in baseline stress beyond $\mathrm{L}_{\max }$ may serve, physiologically, to prevent overdistension of the colon and subsequently ensure that the contractile elements are not stretched beyond the point at which they can exert maximum active stress. This might be of importance in certain pathological conditions which result in over distension of the viscus, particularly as others have shown that stretching smooth muscle preparations to lengths greater than $\mathrm{L}_{\max }$ is associated with an irreversible impairment in the ability to contract. ${ }^{32} 33$ We defined the baseline stress as the minimum stress exerted by the smooth muscle during a cycle of spontaneous contraction. This definition is similar to that of Price et al. ${ }^{17}$ who concluded that the minimum stress exerted by the guinea pig taenia caecum during a cycle of spontaneous contraction was comparable to the passive stress exerted by a 
preparation in which the spontaneous contractions had been arrested with adrenaline. We did not determine the passive properties of human colonic muscle after the abolition of spontaneous contractions and cannot exclude the possibility that the contractile element, as well as connective tissue and non-contractile elements, contributed to the baseline stress.

The absolute values for the maximum active stress of spontaneously-contracting human colonic muscle reported in this paper are not likely to reflect the maximum active stress that this muscle is capable of generating. Gabella ${ }^{34}$ has shown that the active contractile stress exerted by guinea-pig taenia caecum in response to carbachol is greater than the active stress of spontaneous contraction at the same muscle length. Thus, we cannot assume that all the contractile elements of human colonic muscle are completely, or even partially, activated during spontaneous contraction. The maximum active stress exerted by the intertaenial longitudinal muscle layer was considerably greater than that of the taenia. This may reflect either a greater activation of the inter-taenial longitudinal muscle layer during spontaneous contraction or a higher muscle-toconnective tissue ratio in the inter-taenial region; histological assessment of the relative proportion of smooth muscle fibres to collagen and elastin is needed.

In conclusion, this study shows that spontaneous contractions of human colonic muscle are dependent upon length. The human colon appears to consist of two distinct areas, based on the mechanical behaviour of the muscle during spontaneous contraction in vitro, which may be related to their differing embryological origin and function.

The authors are indebted to the surgeons attached to the Department of Surgery, University of Alberta Hospital for the acquisition of colonic tissue. We thank Ms K Clarke for typing the manuscript.

\section{References}

1 Gray H. The large intestine. In: Williams $\mathrm{PH}$, Warwick R. eds. Gray's Anatomy, 36th ed; Vol. 8. Splanchnology. Edinburgh: Churchill Livingstone, 1980: 1350-68.

2 Phillips SF, Devroede GJ. Functions of the large intestine. In: Crane RK, ed. International review of physiology, gastrointestinal physiology III. Baltimore: University Park Press, 1979: 263-90.

3 Meschan I, The large intestine. In: Meschan I, ed. An atlas of normal radiographic anatomy, 2nd ed. The intestine beyond the duodenum; the biliary tract. Philadelphia: W B Saunders Company, 1963: 635-4.

4 Torsoli I, Famorino ML, Crucioli V. The relationships between anatomy and motor activity of the colon. Am J Dig Dis 1968; 13: 462-7.
5 Ellis H. Intestinal obstruction. New York: AppletonCentury-Crofts, 1982.

6 Eastwood MA, Watters DAK, Smith AN. Diverticular disease - is it a motility disorder? Clin Gastroenterol 1982; 11: 545-61.

7 Parks TG, Connell AM. Motility studies in diverticular disease of the colon. Gut 1969; 10: 538-42.

8 Smith AN, Shepherd J, Eastwood MA. Pressure changes after balloon distension of the colon wall in diverticular disease. Gut 1981; 22: 841-4.

9 Connell AM, Frankel H, Gottman L. The motility of the pelvic colon following complete lesions of the spinal cord. Paraplegia 1963; 1: 98-115.

10 Sleisinger MH, Silverberg M. Anatomy and developmental anomalies. In: Sleisinger MH, Fordtran JS, eds. Gastrointestinal disease: pathophysiology, diagnosis, management. Section 7. The colon. Philadelphia: W B Saunders Company, 1978: 1505-23.

11 Truelove SC. Movements of the large intestine. Physiol Res 1966; 46: 457-512.

12 Gordon AR, Siegman MJ. Mechanical properties of smooth muscle. I. Length-tension and force-velocity relations. Am J Physiol 1971; 221: 1243-9.

13 Aberg AKG, Axelsson J. Some mechanical aspects of an intestinal smooth muscle. Acta Physiol Scand 1965; 64: 15-27.

14 Lowy J, Mulvany MJ. Mechanical properties of guinea pig taenia coli muscles. Acta Physiol Scand 1973; 88: 123-6.

15 Price JM, Patitucci PJ, Fung YC. Mechanical properties of resting taenia coli smooth muscle. Am J Physiol 1979; 236: 211-20.

16 Price JM, Patitucci PJ, Fung YC. Mechanical properties of taenia coli smooth muscle in spontaneous contraction. Am J Physiol 1977; 233: 47-55.

17 Duthie HL, Kirk D. Electrical activity of human colonic smooth muscle in vitro. $J$ Physiol 1978; 283: 319-30.

18 Vanasin B, Ustach TJ, Schuster MM. Electrical and motor activity of human and dog colon in vitro. John Hopkins Med J 1974; 134: 201-210.

19 van Merwyk AJ, Duthie HL. Characteristics of human colonic smooth muscle in vitro. In: Christensen J, ed. Gastrointestinal motility. New York: Raven Press, 1980: 473-8.

20 Golenhofen K. Slow rhythms in smooth muscle (minute rhythm). In: Bulbring E, Brading AF, Jones AW, Tomita T. Smooth muscle. Baltimore: Williams and Wilkins 1970: 316-42.

21 Huizinga JD, Diamant NE, El-Sharkawy TY. Electrical basis of contractions in the muscle layers of pig colon. Am J Physiol 1983; 245: 482-91.

22 El-Sharkawy TY. Electrical activities of the muscle layers of the canine colon. J Physiol 1976; 263: 199-213.

23 Christensen J, Caprilli R, Lund GF. Electric slow waves in circular muscle of cat colon. Am J Physiol 1969; 217: 771-6.

24 Kerlin P, Zinsmeister A, Phillips S. Motor responses to food of the ileum, proximal colon, and distal colon of healthy humans. Gastroenterology 1983; 84: 762-70.

25 Bülbring E, Kuriyama $H$. The effect of adrenaline on 
the smooth muscle of guinea-pig taenia coli in relation to the degree of stretch. J Physiol 1963; 169: 198-212.

26 Dobrin PB. Influence of initial length on length-tension relationship of vascular smooth muscle. Am J Physiol 1973; 225: 664-70.

27 Herlihy JT, Murphy RA. Force-velocity and series elastic characteristics of smooth muscle from the pig carotid artery. Circ Res 1974; 34: 461-6.

28 Paul RJ, Peterson JW. Relation between length, isometric force, and $\mathrm{O}_{2}$ consumption rate in vascular smooth muscle. Am J Physiol 1975; 228: 915-22.

29 Uvelius B. Isometric and isotonic length-tension relations and variations in cell length in longitudinal smooth muscle from rabbit urinary bladder. Acta Physiol Scand 1976; 97: 1-12.
30 Meiss RA. Some mechanical properties of cat intestinal muscle. Am J Physiol 1971; 220: 2000-2007.

31 Csapo A, Goodall M. Excitability, length-tension relation and kinetics of uterine muscle contraction in relation to hormonal status. J Physiol 1954; 126: 384-95.

32 Paul RJ. Smooth muscle: mechanochemical energy conversion relations between metabolism and contractility. In: Johnson LR, ed. Physiology of the digestive tract. New York: Raven Press, 1981: 269-88.

33 Peterson JW, Paul RJ. Effects of initial length and active shortening on vascular smooth muscle contractility. Am J Physiol 1974; 227: 1019-24.

34 Gabella G. The force generated by a visceral smooth muscle. J Physiol 1976; 263: 199-213. 